\title{
MILLIMETER IMAGING OF MWC 758: PROBING THE DISK STRUCTURE AND KINEMATICS
}

\author{
Andrea Isella ${ }^{1}$, Antonella Natta ${ }^{2}$, David Wilner ${ }^{3}$, John M. CARPEnter $^{1}$, And LeOnardo Testi ${ }^{4}$ \\ ${ }^{1}$ Department of Astronomy, California Institute of Technology, MC 249-17, Pasadena, CA 91125, USA; isella@ astro.caltech.edu \\ 2 Osservatorio Astrofisico di Arcetri, INAF, Largo E. Fermi 5, 50125 Firenze, Italy \\ ${ }^{3}$ Harvard-Smithsonian Center for Astrophysics, 60 Garden Street, MS 42, Cambridge, MA 02138, USA \\ ${ }^{4}$ European Southern Observatory, Karl Schwarzschild Str. 2, D-85748 Garching, Germany \\ Received 2010 July 16; accepted 2010 October 11; published 2010 December 1
}

\begin{abstract}
We investigate the structure and kinematics of the circumstellar disk around the Herbig Ae star MWC 758 using high-resolution observations of the ${ }^{12} \mathrm{CO}(3-2)$ and dust continuum emission at the wavelengths of 0.87 and $3.3 \mathrm{~mm}$. We find that the dust emission peaks at an orbital radius of about $100 \mathrm{AU}$, while the CO intensity has a central peak coincident with the position of the star. The $\mathrm{CO}$ emission is in agreement with a disk in Keplerian rotation around a $2.0 M_{\odot}$ star, confirming that MWC 758 is indeed an intermediate-mass star. By comparing the observation with theoretical disk models, we derive that the disk surface density $\Sigma(r)$ steeply increases from 40 to 100 AU and decreases exponentially outward. Within $40 \mathrm{AU}$, the disk has to be optically thin in the continuum emission at millimeter wavelengths to explain the observed dust morphology, though our observations lack the angular resolution and sensitivity required to constrain the surface density on these spatial scales. The surface density distribution in MWC 758 disk is similar to that of "transition" disks, though no disk clearing has been previously inferred from the analysis of the spectral energy distribution (SED). Moreover, the asymmetries observed in the dust and $\mathrm{CO}$ emission suggest that the disk may be gravitationally perturbed by a low-mass companion orbiting within a radius of $30 \mathrm{AU}$. Our results emphasize that SEDs alone do not provide a complete picture of disk structure and that high-resolution millimeter-wave images are essential to reveal the structure of the cool disk mid-plane.
\end{abstract}

Key words: planet-disk interactions - protoplanetary disks - submillimeter: planetary systems - techniques: interferometric

Online-only material: color figures

\section{INTRODUCTION}

Millimeter-wave observations of circumstellar disks provide the best tool to measure the distribution and kinematics of the circumstellar material. These observations have for years achieved $1^{\prime \prime}-3^{\prime \prime}$ angular resolution to resolve the disk emission on scales down to an orbital radius of about $\sim 70 \mathrm{AU}$ at the distance of the nearby star-forming regions (e.g., Koerner \& Sargent 1995; Guilloteau \& Dutrey 1998; Kitamura et al. 2002). More recent observations are reaching subarcsecond resolution that is resolving disks on scales of the Kuiper Belt and yielding unprecedented information on the innermost disk regions (see, e.g., Andrews et al. 2009; Brown et al. 2009; Hughes et al. 2009; Isella et al. 2009, 2010; Piétu et al. 2006).

In many cases, millimeter observations confirm the radial distribution of dust and gas inferred from the analysis of the disk spectral energy distribution (hereafter SED). The large majority of pre-main-sequence stars exhibit a flux excess over the stellar photosphere from near-infrared to centimeter wavelengths. This excess arises from a "classical," or primordial, disk that extends from few stellar radii to few hundreds of astronomical units. However, about $10 \%$ of the observed systems (Muzerolle et al. 2010) exhibit a deficit of flux in the near- and mid-infrared compared to a "classical" disk, but have similar level of far-infrared emission (i.e., "transition" disk; Strom et al. 1989). The SED shape suggests that these stars lack the emission from warm dust close to the central star. The lack of warm dust emission may result from several different effects such as the presence of a stellar companion, the formation of a planetary system, disk photoevaporation, magnetorotational instabilities, disk viscosity, and grain growth (Alexander \& Armitage 2007; Chiang \& Murray-Clay 2007; Ireland \& Kraus 2008; Isella et al. 2009; Strom et al. 1989; Tanaka et al. 2005). Subsequent high-resolution millimeterwave images have confirmed that several "transition" disks are indeed dust-depleted within the radius predicted by the SED modeling (Piétu et al. 2006; Brown et al. 2009; Hughes et al. 2009).

The increasing sensitivity and resolution of (sub)millimeterwave interferometers are now providing unexpected insights about the structure of "classical" disks. Subarcsecond observations of AB Aur and RY Tau systems indeed show that these two circumstellar disks may be dust depleted within orbital radii of $70 \mathrm{AU}$ and $13 \mathrm{AU}$, respectively (Piétu et al. 2005; Isella et al. 2010), although no disk clearing has been inferred from the analysis of the SED (Robitaille et al. 2007; Dullemond et al. 2001). It is therefore possible that other "classical" disks may be characterized by a disk surface density that drastically deviates from that inferred from the SED modeling. In particular, since the dust opacity in the infrared is much larger than at millimeter-wavelengths, it is reasonable to assume that a large fraction of "classical" disks might by characterized by partially cleared inner cavities which are not detectable through the analysis of the infrared SED.

In this paper, we discuss the Herbig Ae star MWC 758 (HD 36112), a 3 Myr old pre-main-sequence A5 star located near the edge of the Taurus star-forming region at a distance of $200_{-40}^{+60}$ pc (Perryman et al. 1997; van den Ancker et al. 1998). Previous investigations based on the SED modeling and on spatially resolved interferometric observations suggested that 

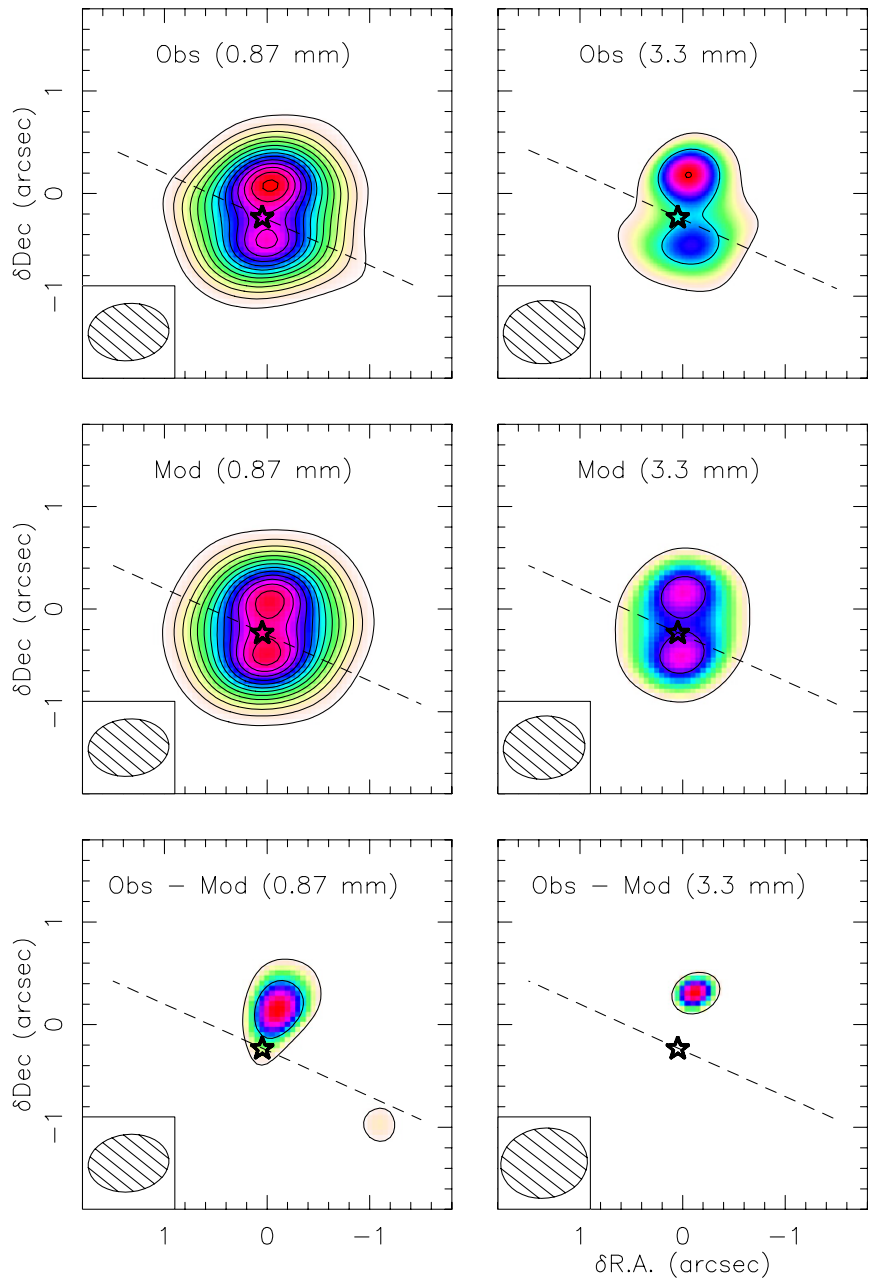

Figure 1. Observations, best-fitting models, and residual maps of the dust continuum emission observed toward MWC 758 by SMA at $0.87 \mathrm{~mm}$ (left column) and by CARMA at $3.3 \mathrm{~mm}$ (right columns). The $1 \sigma$ noise levels are 2.1 and $0.25 \mathrm{mJy}^{-1}$ beam $^{-1}$ in SMA and CARMA observations, respectively. Contours are plotted every $3 \sigma$. The symbol " $\star$ " marks the position of MWC 758 after correcting for the proper motion as described in Section 3 . The dashed line shows the orientation of the disk major axis derived from the $\mathrm{CO}$ observations as discussed in Section 3 .

(A color version of this figure is available in the online journal.)

MWC 758 is surrounded by a classical, fully flared rotating disk that extends from few stellar radii (Isella et al. 2008; Eisner et al. 2004) up to an outer radius of $385 \pm 26 \mathrm{AU}^{6}$ (Chapillon et al. 2008). At $1.3 \mathrm{~mm}$, dust continuum emission observed at $2^{\prime \prime}$ angular resolution is centrally peaked and more compact than the ${ }^{12} \mathrm{CO}(2-1)$ emission (Chapillon et al. 2008). This difference in radial extension between dust and gas emission is similar to that found in other circumstellar disks (see, e.g., Piétu et al. 2005; Isella et al. 2007) and can be explained with an exponential decrease of the disk surface density in the outermost disk regions (Hughes et al. 2008; Isella et al. 2010).

Here we present new images of the millimeter-wave dust continuum and ${ }^{12} \mathrm{CO}$ emission toward MWC 758 characterized by an angular resolution of 0!7, which is three times better than previous observations. Our observations resolve the inner disk into two peaks in the continuum emission, strongly suggesting

\footnotetext{
6 Note that Chapillon et al. and Eisner et al. assume a stellar distance of 140 and $150 \mathrm{pc}$, respectively. The radii quoted from these two papers have been therefore rescaled for the distance of $200 \mathrm{pc}$.
}

that the disk surface density decreases within an orbital radius of about $100 \mathrm{AU}$. Similar to the cases of AB Aur and RY Tau, the MWC 758 inner disk may be dust-depleted with respect to a power-law surface density profile, though no disk clearing has been inferred from the analysis of the SED.

The observations, presented in Sections 2 and 3, are compared with theoretical disk models to constrain the surface density profile of MWC 758 disk. The results are presented in Section 4. The discussion and conclusions follow in Section 5.

\section{OBSERVATIONS AND DATA REDUCTION}

\subsection{SMA Observations}

The MWC 758 system was observed at $345 \mathrm{GHz}$ with the Submillimeter Array ${ }^{7}$ (SMA) on Mauna Kea, Hawaii, on 2008 January 28 . The array was in the extended configuration, and the seven available antennas provided baseline lengths from 31 to $225 \mathrm{~m}$. The atmospheric transparency was excellent through the track, with an opacity of 0.04 at $225 \mathrm{GHz}$ measured at the nearby Caltech Submillimeter Observatory. The correlator provided $2 \mathrm{GHz}$ of bandwidth in two sidebands separated by $10 \mathrm{GHz}$, with the upper sideband centered near the $\mathrm{CO}$ $J=3-2$ line at $345.796 \mathrm{GHz}$. This line was observed at a spectral resolution $0.18 \mathrm{~km} \mathrm{~s}^{-1}$ in a single correlator "chunk" spanning $104 \mathrm{MHz}$. The primary flux calibrator was Titan, and the passband calibrators were the quasars $3 \mathrm{C} 273$ and $3 \mathrm{C} 111$. Complex gain calibration was carried out with observations of the quasar J0530+135 (1.75 Jy) interleaved with MWC 758. Data were obtained for MWC 758 over the hour angle range -3.2 to +4.7 . All of the data editing and calibration were performed using standard routines in the MIR software package for IDL. The resulting map of the dust continuum emission is shown in Figure 1. Applying natural weighting to the data, we achieve a noise level of $2.1 \mathrm{mJy}^{-1}$ beam $^{-1}$ and synthesized beam FWHM of $0.76 \times 0$ 0'56. Figure 2 shows the $\mathrm{CO}$ emission in the velocity range between 2.94 and $8.93 \mathrm{~km} \mathrm{~s}^{-1}$, as obtained by averaging the observations every two channels. The resulting noise level is $83 \mathrm{mJy}^{\text {beam }}{ }^{-1}$.

\subsection{CARMA Observations}

The dust thermal emission toward MWC 758 was observed on 2009 January using the Combined Array for Research in Millimeter-wave Astronomy ${ }^{8}$ (CARMA) located at the altitude of 7200 feet in the Inyo mountains of eastern California. The array was in the extended B configuration, which provides baselines between 80.9 and $946.3 \mathrm{~m}$. The observations were obtained at the local oscillator frequency of $97.5 \mathrm{GHz}(\lambda \sim$ $3.3 \mathrm{~mm}$ ). The CARMA correlator was configured with six bands, each of which was configured to $468 \mathrm{MHz}$ bandwidth to provide maximum continuum sensitivity. No molecular emission line was observed with CARMA during this track. The passband shape was calibrated by observing $3 \mathrm{C} 454.3$ and the flux calibration was set by observing Uranus. The

\footnotetext{
7 The Submillimeter Array is a joint project between the Smithsonian Astrophysical Observatory and the Academia Sinica Institute of Astronomy and Astrophysics and is funded by the Smithsonian Institution and the Academia Sinica.

8 Support for CARMA construction was derived from the Gordon and Betty Moore Foundation, the Kenneth T. and Eileen L. Norris Foundation, the James S. McDonnell Foundation, the Associates of the California Institute of Technology, the University of Chicago, the states of California, Illinois, and Maryland, and the National Science Foundation. Ongoing CARMA development and operations are supported by the National Science Foundation under a cooperative agreement and by the CARMA partner universities.
} 

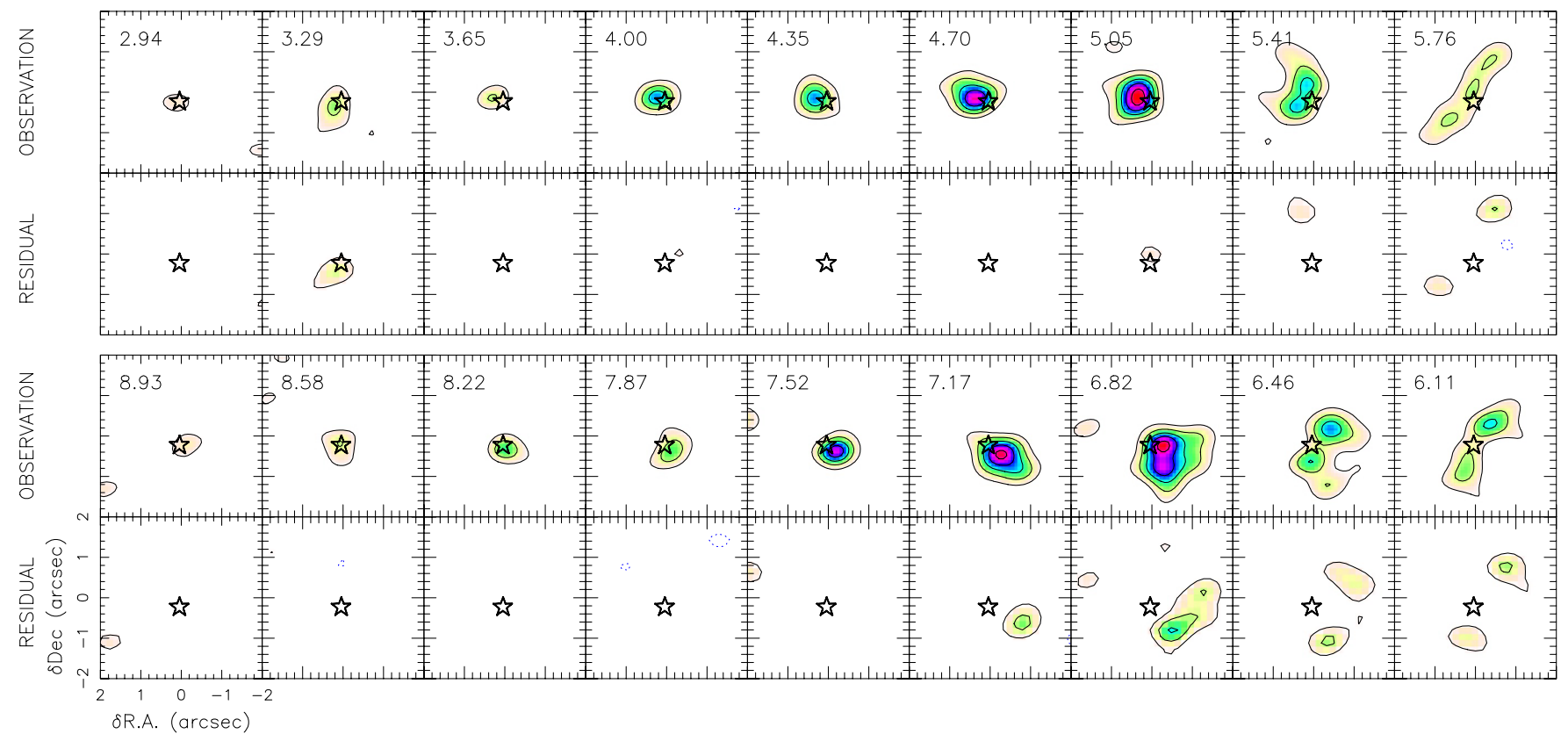

Figure 2. Channel maps for the ${ }^{12} \mathrm{CO} J=3-2$ line emission observed toward MWC 758 in the velocity range between 2.94 and $8.93 \mathrm{~km} \mathrm{~s}^{-1}$. The contours are spaced by $3 \sigma=0.25 \mathrm{Jy} \mathrm{beam}^{-1}$. The figure also shows the residuals obtained by subtracting the best-fitting model CO emission from the observations. The symbol " $\star$ " marks the position of the central star after correcting for the proper motion.

(A color version of this figure is available in the online journal.)
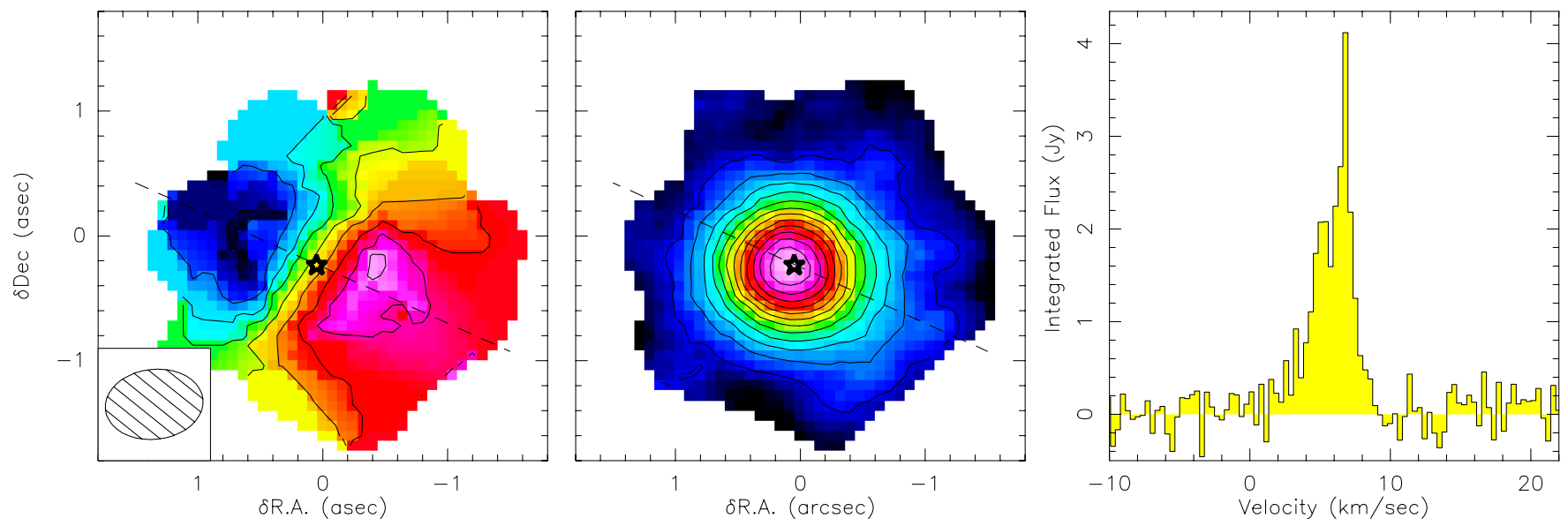

Figure 3. Left panel: ${ }^{12} \mathrm{CO} J=3-2$ velocity gradient across MWC 758 circumstellar disk. Red and blue colors indicate the redshifted and blueshifted disk velocities, respectively. Velocity contours start at $4.7 \mathrm{~km} \mathrm{~s}^{-1}$ and are spaced by $0.3 \mathrm{~km} \mathrm{~s}^{-1}$. The dashed line indicates the position angle of the apparent disk major axis, which is rotated by $65^{\circ}$ to east from north. Central panel: ${ }^{12} \mathrm{CO} \mathrm{J}=3-2$ integrated intensity map. Right panel: ${ }^{12} \mathrm{CO} J=3-2$ spectrum obtained integrating over the region shown in the intensity map.

(A color version of this figure is available in the online journal.)

quasar J0510+180 was observed every 15 minutes to correct for atmospheric and instrumental effects. The data reduction and the image reconstruction were obtained using the MIRIAD software package. Figure 1 shows the resulting map, which is characterized by an rms noise of $0.25 \mathrm{mJy}^{\text {beam }}{ }^{-1}$ and a synthesized beam FWHM of 0 ' $80 \times 0$ 0'62 when natural weighting is applied to the data.

\section{RESULTS}

Figures 1-3 show the dust and CO emission toward MWC 758 as observed by pointing the telescope at the International Celestial Reference System coordinates of the star at the epoch J2000, R.A. $=05^{\mathrm{h}} 30^{\mathrm{m}} 27^{\varsigma} .530( \pm 12.89$ mas $)$ and decl. $=+25^{\circ} 19^{\prime} 57^{\prime \prime} .08( \pm 5.57$ mas $)$. The position of the star at the date of the observation is shown by the symbol " $\star$ " and is calculated by applying a proper motion of $\delta$ (R.A.) $=5.23 \pm$

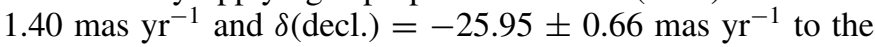
J2000 coordinates (both the stellar coordinates and the proper motion are from Perryman et al. 1997). The uncertainties on the stellar position resulting both from the Hipparcos astrometry and proper motion uncertainties are smaller than the size of symbol itself.

The dust emission at both 0.87 and $3.3 \mathrm{~mm}$ is characterized by two peaks which are symmetric with respect to the position of the star and are separated by about 0.7 , or $140 \mathrm{AU}$ at the distance of $200 \mathrm{pc}$. At the wavelength of $0.87 \mathrm{~mm}$, the northern and southern peaks have fluxes of 89 and $77 \mathrm{mJy}^{\text {beam }}{ }^{-1}$, 


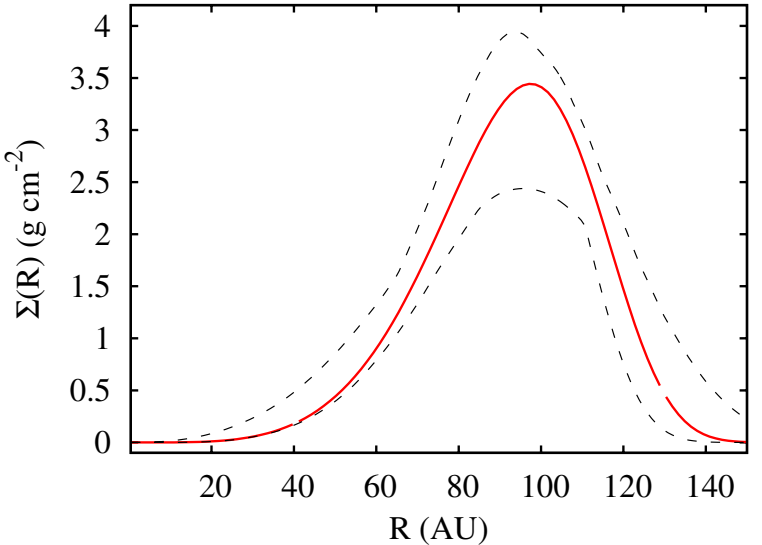

Figure 4. Solid red line shows the best-fitting model solution for the disk surface density $\Sigma(r)$ obtained by analyzing the spatially resolved observations of the dust and $\mathrm{CO}$ emission at both 0.87 and $3.3 \mathrm{~mm}$. The dashed lines indicate the $3 \sigma$ uncertainty range. Given the angular resolution and sensitivity of our observations, $\Sigma(r)$ is constrained only between 40 and $130 \mathrm{AU}$. Within $40 \mathrm{AU}$, $\Sigma(r)$ can significantly deviate from the profile shown in the figure, though the disk must remain optically thin at both 0.87 and $3.3 \mathrm{~mm}$ to explain the two peaks observed in the dust emission.

(A color version of this figure is available in the online journal.)

respectively. A similar flux ratio between the two peaks is measured at $3.3 \mathrm{~mm}$, suggesting that the observed morphology corresponds to an asymmetric dust emission between the two sides of the disk. By integrating over a circle with a radius of $1^{\prime \prime} .5$ centered at the position of the star, we measure fluxes of 217 $\pm 40 \mathrm{mJy}$ and $4.9 \pm 1.7 \mathrm{mJy}$ at 0.87 and $3.3 \mathrm{~mm}$, respectively, where the errors account for both the thermal noise and absolute calibration uncertainties. The integrated fluxes are in agreement with the values measured at $2^{\prime \prime}$ angular resolution by Chapillon et al. (2008) suggesting that the dust emission is all coming from the spatial scales probed by our observations. Assuming that the disk millimeter emission follows a power law $F(v) \propto v^{\alpha}$, we derive a spectral index $\alpha_{1-3 \mathrm{~mm}}=3.0$.

Figure 2 shows the ${ }^{12} \mathrm{COJ}=3-2$ channel maps in the velocity range between 2.94 and $8.93 \mathrm{~km} \mathrm{~s}^{-1}$. The $\mathrm{CO}$ emission is spatially resolved and shows a velocity pattern typical of an inclined rotating disk. The first moment of the $\mathrm{CO}$ emission, i.e., the velocity gradients across the disk, indicates that the western and eastern sides of the disk are, respectively, redshifted and blueshifted with respect to the relative velocity of the star (see the left panel of Figure 3). The velocity gradient also suggests a position angle of the disk major axis of about $65^{\circ}$ as measured from north to east. The central panel of Figure 3 shows the spectrally integrated $\mathrm{CO}$ emission. In contrast to what is observed in the dust continuum, the $\mathrm{CO}$ emission has a single peak coincident with the position of the star. The $\mathrm{CO}$ emission is observed up to a distance of $1^{\prime \prime} .3$ in the redshifted side of the disk and only to about $1^{\prime \prime}$ on the other side. The line integrated flux is $12.9 \mathrm{Jy} \mathrm{km} \mathrm{s}^{-1}$. This value is about $14 \%$ smaller than that measured in single dish observations (Dent et al. 2005), suggesting that part of the $\mathrm{CO}$ emission is resolved out in the interferometric observations. As a consequence, the observed asymmetry in the $\mathrm{CO}$ emission might be due to the lack of short baselines in the SMA observations instead of to a real asymmetry in the gas temperature or distribution. Finally, the spatially integrated $\mathrm{CO}$ spectrum (the right panel of Figure 3 ) has a double peak profile centered at the velocity of $v_{\mathrm{lsr}}=6.0 \mathrm{~km} \mathrm{~s}^{-1}$. The line emission is observed in the velocity range $v_{\mathrm{lsr}} \pm 4 \mathrm{~km} \mathrm{~s}^{-1}$ with a brighter flux in the redshifted half of the disk.
To date, the different morphologies observed in the dust and gas emission toward MWC 758 represent a unique case among the sample of circumstellar disks spatially resolved with millimeter-wave interferometers. Our observations suggest that the optical depth of the dust continuum emission decreases within a radius of 70-100 AU from the star. Despite that, the disk remains gas-rich down to at least a distance of 30 AU to explain the centrally peaked $\mathrm{CO}$ emission. In addition, the SED of MWC 758 shows a flux excess above the stellar photosphere between near- and far-infrared wavelengths typical of optically thick flaring disk extending to few stellar radii (see Figure 5). The following section is devoted to reconciling all these observations in the framework of existing disk models in order to constrain the radial distribution of the circumstellar material around MWC 758.

\section{DATA ANALYSIS}

\subsection{Disk Models}

To analyze the data, we adopt the disk model discussed in Isella et al. (2007, 2009). In brief, we compute the radial dust temperature profile following the "two-layer" approximation of Chiang \& Goldreich (1997), where the disk is characterized by a warm surface layer and a cooler interior. Both temperatures are calculated as a function of the orbital radius by iterating on the vertical disk structure (see Dullemond et al. 2001). The disk is in hydrostatic equilibrium between the gas pressure and the stellar gravity, which leads to a flared geometry with the opening angle increasing with the distance from the central star.

The dust opacity is calculated by assuming an interstellar grain composition (Pollack et al. 1994) and a particle size distribution between a minimum and a maximum value $a_{\text {min }}$ and $a_{\max }$ according to $n(a) \propto a^{-q}$. We fix $a_{\min }=0.05 \mu \mathrm{m}$ and vary $a_{\max }$ and $q$ to reproduce the spectral index $\alpha$ of the millimeter disk emission. For the sake of simplicity, we assume that the dust opacity is constant throughout the disk (although see Birnstiel et al. 2010).

The radial distribution of the circumstellar material follows the similarity solution for the disk surface density of a viscous Keplerian disk (Lynden-Bell \& Pringle 1974) expressed by

$$
\Sigma(r, t)=\Sigma_{t}\left(\frac{r_{t}}{r}\right)^{\gamma} \times \exp \left\{-\frac{1}{2(2-\gamma)}\left[\left(\frac{r}{r_{t}}\right)^{(2-\gamma)}-1\right]\right\},
$$

where the characteristic radius $r_{t}, \gamma$, and the surface density normalization $\Sigma_{t}$ are free parameters of the model, as well as the disk inclination and position angle.

From the derived dust density, temperature, and opacity, we calculate the disk SED and synthetic disk images in the dust continuum at $0.87 \mathrm{~mm}$ and $3.3 \mathrm{~mm}$ using the radiative transfer solution discussed in Dullemond et al. (2001). The synthetic disk images are then Fourier transformed and sampled at the appropriate positions on the $(u, v)$ plane corresponding to our CARMA and SMA observations.

The $\mathrm{CO}$ emission is calculated assuming that rotational levels are thermalized. The $\mathrm{CO}$ temperature is parameterized as a function of the radius as $T_{\mathrm{CO}}(r)=T_{\mathrm{CO}}\left(r_{0}\right)\left(r_{0} / r\right)^{\zeta}$, where $T_{\mathrm{CO}}\left(r_{0}\right)$ and $\zeta$ are free parameters and $r_{0}=50 \mathrm{AU}$. The $\mathrm{CO}$ density is then calculated by assuming that the dust and the gas are well mixed with a gas-to-dust ratio of 100 . The adopted $\mathrm{CO}$ abundance is ${ }^{12} \mathrm{CO} / \mathrm{H}_{2}=7.0 \times 10^{-5}$. Finally, the $\mathrm{CO}$ channel maps are calculated in the velocity range probed by our observations by assuming that the emission originates 
Table 1

Best-fitting Model Parameters for the Dust and CO Millimeter Emission Observed Toward MWC 758

\begin{tabular}{l}
\hline \hline Disk orientation: \\
Inclination $=21^{\circ} \pm 2^{\circ}, \mathrm{P} . \mathrm{A} .=65^{\circ} \pm 7^{\circ}$ \\
\hline Surface density $\Sigma(R):$ \\
$\gamma=-4.1 \pm 0.3, R_{t}=69 \pm 3 \mathrm{AU}, \Sigma_{t}=1.5 \pm 0.1 \mathrm{~g} \mathrm{~cm}^{-2}$ \\
\hline $\mathrm{CO}$ temperature $T(\mathrm{CO}):$ \\
$T_{\mathrm{CO}}(50 \mathrm{AU})=53 \pm 4 \mathrm{~K}, \zeta=-0.1 \pm 0.2$ \\
$M_{\star}=2.0 \pm 0.2 M_{\odot}$ \\
\hline Grain size distribution $n(a):$ \\
$a_{\max }=1 \mathrm{~cm}, q=3.5$ \\
\hline
\end{tabular}

Note. The quoted uncertainties correspond to the $1 \sigma$ uncertainty level.

from an inclined Keplerian disk. Both the disk inclination and the mass of the central star are free parameters of the model (see the Appendix of Isella et al. 2007, for more details on the $\mathrm{CO}$ emission model). As for the case of the dust emission, the synthetic CO channel maps are then Fourier transformed and compared to the measured complex visibilities.

The best-fitting models for both the dust and gas emission, and the uncertainties on the model free parameters, are then obtained by $\chi^{2}$ minimization carried out using the Markov Chain Monte Carlo method discussed in Isella et al. (2009).

\subsection{Results of the Model Fitting}

A satisfactory fit of the spatially resolved dust and $\mathrm{CO}$ emission, as well as of the SED between far-infrared and (sub)millimeter wavelengths, is obtained with the disk model presented in Table 1 . The disk surface density is mainly constrained by the optically thin dust emission at 0.87 and $3.3 \mathrm{~mm}$ and is characterized by $\gamma=-4.1$ and $r_{t}=69 \mathrm{AU}$. As shown in Figure 4, $\Sigma(r)$ has a "bell" shape that reaches the maximum value of $3.3 \mathrm{~g} \mathrm{~cm}^{-2}$ at the radius of $100 \mathrm{AU}$. For $r \ll r_{t}$, the surface density is proportional to $r^{4}$, suggesting that a steep radial density profile is required to explain the two peaks observed in the dust emission. For $r>100 \mathrm{AU}, \Sigma(r)$ fades exponentially as prescribed by Equation (1). Given the angular resolution and sensitivity of our observations, we can constrain the surface density only between 40 and 130 AU. In this range, the disk is optically thin to the dust continuum emission at both 0.87 and $3.3 \mathrm{~mm}$. Within $40 \mathrm{AU}$, the radial profile of $\Sigma(r)$ might significantly deviate from that shown in Figure 4. However, the disk must remain optically thin to the dust continuum millimeter-wave emission down to at least few AU in order to reproduce the two peaks shown in Figure 1.

Radially integrating the surface density, we obtain a disk mass of $0.01 M_{\odot}$. This result is affected by a large error due to the uncertainties on the dust opacity and on the gas-to-dust ratio. In particular, the analysis of the ${ }^{12} \mathrm{CO}(2-1)$ and $(1-0)$ line emission suggests that the gas-to-dust ratio in MWC 758 disk might be smaller than 100 (Chapillon et al. 2008), leading therefore to a smaller disk mass.

Despite the steep surface density profile, the ${ }^{12} \mathrm{CO}(3-2)$ emission remains optically thick down to a radius of about $20 \mathrm{AU}$. As a consequence, the $\mathrm{CO}$ emission does not constrain the disk surface density but it provides a measure of the $\mathrm{CO}$ temperature in the form $T_{\mathrm{CO}}(r) \simeq 53 \mathrm{~K} \times(50 \mathrm{AU} / r)^{-0.1}$. Note that the ${ }^{12} \mathrm{CO}(3-2)$ emission remains optically thick even if the $\mathrm{CO}$ is largely depleted with respect to the standard abundance. In particular, assuming a $\mathrm{CO}$ abundance of $10^{-6}$ (Chapillon et al. 2008) we calculate a mean optical depth of about $10^{3}$. The almost radially constant profile of the $\mathrm{CO}$ temperature is in good agreement with the value $\zeta=0.05 \pm 0.2$ derived from the analysis of the ${ }^{12} \mathrm{CO}(2-1)$ emission line by Chapillon et al. (2008). In the assumption that the disk is in Keplerian rotation, we derive a disk inclination of $22^{\circ}$ and a dynamical mass for the central star of $2.0 M_{\odot}$, similar to the value of $2.3 M_{\odot}$ derived from the H-R diagram using stellar evolutionary tracks (Manoj et al. 2006).

The residuals obtained by subtracting the best-fitting model to the observations reveal asymmetries in the MWC 758 disk. In the case of the dust emission (see Figure 1), our disk model fails to reproduce the flux measured in the northern side of the disk at both 0.87 and $3.3 \mathrm{~mm}$. From the intensity of the residuals, we calculate that the dust density, opacity, and/or temperature in the northern side of the disk might be $20 \%$ higher than the southern side. Figure 2 shows the residuals for the CO channel maps in the velocity range between 2.94 and $6.11 \mathrm{~km} \mathrm{~s}^{-1}$. Most of the significative structures appear in low-velocity channels of the redshifted side of the disk between 6.11 and $7.11 \mathrm{~km} \mathrm{~s}^{-1}$. Since the $\mathrm{CO}$ emission is almost completely optically thick, this suggests that the $\mathrm{CO}$ temperature might be higher in this side or that the $\mathrm{CO}$ extends to slightly larger distances from the central star. Note, however, that the CO asymmetry might also be due to the lack of short spatial frequency in SMA observations as discussed in Section 3.

Figure 5 shows the comparison between the observed SED and the SED of the best-fitting model to the millimeter data. At millimeter wavelengths, a good agreement with the observations is obtained with a grain size distribution slope $q=3.5$ and a maximum grain size $a_{\max }=1 \mathrm{~cm}$. Fitting the dust opacity $k(\lambda)$ between 1.3 and $2.8 \mathrm{~mm}$ with a power law $k(\lambda) \propto \lambda^{-\beta}$, we obtain $\beta=1.35$, which is a factor of two higher than the mean value measured in T Tauri stars but in agreement with those found in other Herbig Ae stars (see, e.g., Ricci et al. 2010; Natta et al. 2004).

The good agreement between the model and the observation extends down to a wavelengths of about $20 \mu \mathrm{m}$. This suggests that the disk has a flared structure in the region that emits most of the mid- and far-infrared emission, namely between 20 and $110 \mathrm{AU}$. At shorter wavelengths, the emission of the best-fitting model is much lower than what observed toward MWC 758. This is due to the fact that for $\Sigma(r) \propto r^{4}$, the dust density within few AU from the central star is lower than $10^{-6} \mathrm{~g} \mathrm{~cm}^{-2}$ and its near-infrared emission is negligible with respect to the stellar photospheric emission. The observed near- 


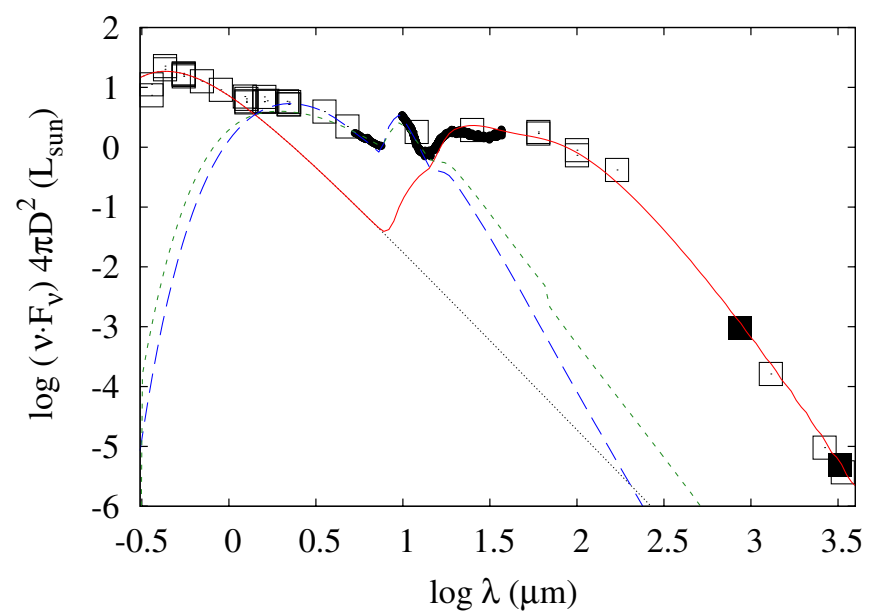

Figure 5. SED of MWC 758. Photometric data from the literature are shown with empty squares (Eisner et al. 2004; Malfait et al. 1998; Beskrovnaya et al. 1999; Cutri et al. 2003; Beichman et al. 1988; Elia et al. 2005; Chapillon et al. 2008). The thick black line shows the observed IRS spectrum from the Spitzer archive. The filled squares show our new measurements at 0.8 and $3.3 \mathrm{~mm}$. Optical- and near-infrared data were de-reddened adopting $A_{V}=0.2$ (van den Ancker et al. 1998). The dotted black line shows the stellar photosphere modeled using a blackbody emission with a temperature $T_{\text {eff }}=8200 \mathrm{~K}, L_{\star}=22 L_{\odot}$, and a distance of $200 \mathrm{pc}$ (van den Ancker et al. 1998). The solid red line shows the SED for the best-fitting model discussed in Section 3. The long-dashed blue and short-dashed green curves show the SED of an optically thick and thin inner disk model, as discussed in Section 4.2.

(A color version of this figure is available in the online journal.)

infrared excess therefore suggests the presence of material with temperature between 1000 and $2000 \mathrm{~K}$ in excess to what was predicted by our model.

To reconcile the near-infrared SED with our millimeter-wave observations, we propose two different scenarios. In the first case, the near-infrared emission is coming from an optically thick disk extending between 0.05 and 5 AU. This model comprises a gaseous disk between 0.05 and 0.1 AU, a "puffedup" inner rim of the dusty disk between 0.4 and 0.5 AU (see, e.g., Isella \& Natta 2005), and a flaring disk between 0.5 and 5 AU. As discussed in Isella et al. (2008), this model explains the spectro-interferometric observations of the H- $(1.6 \mu \mathrm{m})$ and K-band $(2.2 \mu \mathrm{m})$ disk emission (see also Benisty et al. 2010). The resulting SED, which is indicated by the shortdashed green line in Figure 5, provides a good description of the near-infrared emission and of the $10 \mu \mathrm{m}$ silicate feature. Alternatively, the infrared SED can be explained with the presence of an optically thin layer of dust surrounding the star (Vinković et al. 2006; Mulders et al. 2010). In this case, we calculate that a ring of dust extending between 0.15 and $0.8 \mathrm{AU}$ and characterized by an optical depth for the stellar radiation along the direction perpendicular to the disk mid-plane of 0.4 is required to reproduce the disk emission (see the long-dashed curve in Figure 5). For the assumed dust opacity, this model requires a minimum surface density of $0.01 \mathrm{~g} \mathrm{~cm}^{2}$ or a total mass of $2 \times 10^{-9} M_{\odot}$. Note that neither of these models will produce a significant dust and $\mathrm{CO}$ emission at millimeter wavelengths.

To summarize, assuming the similarity solution for a Keplerian viscous disk, we find strong evidence that the surface density of the MWC 758 disk increases as $r^{4}$ between 40 and $100 \mathrm{AU}$ from the central star and decreases exponentially outward. However, this solution for the surface density is not unique and observations with higher angular resolution are required to discriminate between different models. We note, for example, that a discontinuity in the surface density at about 80 AU from the central star might provide an equally good fit with a different step gradient. Within $40 \mathrm{AU}, \Sigma(r)$ is constrained by the analysis of the SED. We calculate that a minimum surface density value of $10^{-2} \mathrm{~g} \mathrm{~cm}^{-2}$ between 0.15 and $0.8 \mathrm{AU}$ is required to reproduce the observed near- and mid-infrared emission. Probing these spatial scales requires high sensitivity 0 .' 1 resolution observations that will become possible with the Atacama Large Millimeter Array (ALMA).

\section{DISCUSSION AND CONCLUSIONS}

The surface density profile in MWC 758 is similar to that measured in the transitional disk around the $\mathrm{T}$ Tauri star LkCa 15. In this case, $\Sigma(r)$ peaks at about $50 \mathrm{AU}$ from the central star (Piétu et al. 2006; Espaillat et al. 2007) and a minimum mass of $1 \times 10^{-11} M_{\odot}$ within 5 AU from the central star is required to produce the observed near-infrared emission (Mulders et al. 2010). Similarly to what suggested for LkCa15, the shape of the surface density in MWC 758 may be due to the disk clearing operated by a low-mass stellar companion or by one or more giant planets. To this regard, we note that recent spectroastrometric observations of MWC 758 exclude the presence of a stellar companion more massive than about $0.9 M_{\odot}$ orbiting between 0.15 and 0 '. 3 , or 30 and $60 \mathrm{AU}$ for the assumed stellar distance of $200 \mathrm{pc}$ (Wheelwright et al. 2010; H. E. Wheelwright 2010, private communication). The nature of a possible companion of MWC 758 remains therefore uncertain.

The asymmetries observed in the dust and $\mathrm{CO}$ emission support the hypothesis that the disk may be gravitationally perturbed by a massive body. The asymmetric $\mathrm{CO}$ emission can be due to a warped optically thick inner disk, i.e., more inclined than the outer disk, so that the $\mathrm{CO}$ in northwest side of the disk is partially shielded by the inner disk from the stellar radiation. This would result in a lower gas temperature and consequently lower emission with respect to the other side of the disk (see, e.g., Panić et al. 2010). Indeed, we note that the inclination of the inner disk derived from near-infrared interferometric observations is of about $40^{\circ} \pm 10^{\circ}$ (Isella et al. 2006, 2008; Eisner et al. 2004), which is larger than the $21^{\circ} \pm 2^{\circ}$ derived in this work.

Other mechanisms, which are in principle able to evacuate the inner disk, do not seem to be consistent with the observations. Photoevaporation and magnetorotational instability models predict completely cleared inner disks and surface density profiles at the inner disk radius much steeper than $r^{4}$ (Alexander \& Armitage 2007; Chiang \& Murray-Clay 2007). In addition, the surface density within few AU is much larger than that predicted by the viscous solution for the surface density that best fits the millimeter data. This fact excludes that $\Sigma(r)$ is shaped only by the radial profile of the disk viscosity (see the discussion in Isella et al. 2009). Low dust opacity caused by grain growth has also been suggested to explain inner holes observed in the continuum emission at millimeter wavelengths (Tanaka et al. 2005). In the case of MWC 758, the dust opacity should decrease by at least two orders of magnitude to explain the observations. This would require a grain size distribution flatter than what typically assumed (i.e., $n(a) \propto a^{-q}$ with $q<3$ ) and a steep radial decrease of the maximum grain (i.e., from $10 \mathrm{~cm}$ at $10 \mathrm{AU}$ to $10 \mu \mathrm{m}$ at $100 \mathrm{AU})$.

In conclusion, our observations show that the disk around MWC 758 is characterized by a steeply increasing surface density between 40 and 100 AU from the central star. Given the infrared SED and the asymmetries observed in the gas and dust 
emission, we conclude that the surface density might be shaped by the presence of stellar or planetary size companion orbiting within $30 \mathrm{AU}$. The fact that MWC 758 was previously labeled as "classical" disk emphasizes that SEDs alone do not provide a complete picture of disk structure and that high-resolution millimeter-wave images are essential to reveal the structure of the cool disk mid-plane.

We thank the OVRO/CARMA staff and the CARMA observers for their assistance in obtaining the data. We acknowledge support from the Owens Valley Radio Observatory, which is supported by the National Science Foundation through grant AST 05-40399. This work was performed in part under contract with the Jet Propulsion Laboratory (JPL) funded by NASA through the Michelson Fellowship Program. JPL is managed for NASA by the California Institute of Technology.

\section{REFERENCES}

Alexander, R. D., \& Armitage, P. J. 2007, MNRAS, 375, 500

Andrews, S. M., Wilner, D. J., Hughes, A. M., Qi, C., \& Dullemond, C. P. 2009, ApJ, 700, 1502

Beichman, C. A., Neugebauer, G., Habing, H. J., Clegg, P. E., \& Chester, T. J. (ed.) 1988, Infrared Astronomical Satellite (IRAS) Catalogs and Atlases, Vol. 1: Explanatory Supplement

Benisty, M., et al. 2010, A\&A, 511, A74

Beskrovnaya, N. G., et al. 1999, A\&A, 343, 163

Birnstiel, T., et al. 2010, A\&A, 516, L14

Brown, J. M., Blake, G. A., Qi, C., Dullemond, C. P., Wilner, D. J., \& Williams, J. P. 2009, ApJ, 704, 496

Chapillon, E., Guilloteau, S., Dutrey, A., \& Piétu, V. 2008, A\&A, 488, 565

Chiang, E. I., \& Goldreich, P. 1997, ApJ, 490, 368

Chiang, E., \& Murray-Clay, R. 2007, Nature Phys., 3, 604

Cutri, R. M., et al. (ed.) 2003, The IRSA 2MASS All-Sky Point Source Catalog, The NASA/IPAC Infrared Science Archive, http://irsa.ipac.caltech.edu/ applications/Gator

Dent, W. R. F., Greaves, J. S., \& Coulson, I. M. 2005, MNRAS, 359, 663

Dullemond, C. P., Dominik, C., \& Natta, A. 2001, ApJ, 560, 957

Eisner, J. A., Lane, B. F., Hillenbrand, L. A., Akeson, R. L., \& Sargent, A. I. 2004, ApJ, 613, 1049
Elia, D., Strafella, F., Campeggio, L., Maiolo, B., \& Pezzuto, S. 2005, New Astron., 10, 545

Espaillat, C., Calvet, N., D’Alessio, P., Hernández, J., Qi, C., Hartmann, L., Furlan, E., \& Watson, D. M. 2007, ApJ, 670, L135

Guilloteau, S., \& Dutrey, A. 1998, A\&A, 339, 467

Hughes, A. M., Wilner, D. J., Qi, C., \& Hogerheijde, M. R. 2008, ApJ, 678, 1119

Hughes, A. M., et al. 2009, ApJ, 698, 131

Ireland, M. J., \& Kraus, A. L. 2008, ApJ, 678, L59

Isella, A., Carpenter, J. M., \& Sargent, A. I. 2009, ApJ, 701, 260

Isella, A., Carpenter, J. M., \& Sargent, A. I. 2010, ApJ, 714, 1746

Isella, A., \& Natta, A. 2005, A\&A, 438, 899

Isella, A., Tatulli, E., Natta, A., \& Testi, L. 2008, A\&A, 483, L13

Isella, A., Testi, L., \& Natta, A. 2006, A\&A, 451, 951

Isella, A., Testi, L., Natta, A., Neri, R., Wilner, D., \& Qi, C. 2007, A\&A, 469, 213

Kitamura, Y., Momose, M., Yokogawa, S., Kawabe, R., Tamura, M., \& Ida, S. 2002, ApJ, 581, 357

Koerner, D. W., \& Sargent, A. I. 1995, AJ, 109, 2138

Lynden-Bell, D., \& Pringle, J. E. 1974, MNRAS, 168, 603

Malfait, K., Bogaert, E., \& Waelkens, C. 1998, A\&A, 331, 211

Manoj, P., Bhatt, H. C., Maheswar, G., \& Muneer, S. 2006, ApJ, 653, 657

Mulders, G. D., Dominik, C., \& Min, M. 2010, A\&A, 512, A11

Muzerolle, J., Allen, L. E., Megeath, S. T., Hernández, J., \& Gutermuth, R. A. 2010, ApJ, 708, 1107

Natta, A., Testi, L., Neri, R., Shepherd, D. S., \& Wilner, D. J. 2004, A\&A, 416, 179

Panić, O., van Dishoeck, E. F., Hogerheijde, M. R., Belloche, A., Güsten, R., Boland, W., \& Baryshev, A. 2010, A\&A, 519, 210

Perryman, M. A. C., et al. 1997, A\&A, 323, L49

Piétu, V., Dutrey, A., Guilloteau, S., Chapillon, E., \& Pety, J. 2006, A\&A, 460, L43

Piétu, V., Guilloteau, S., \& Dutrey, A. 2005, A\&A, 443, 945

Pollack, J. B., Hollenbach, D., Beckwith, S., Simonelli, D. P., Roush, T., \& Fong, W. 1994, ApJ, 421, 615

Ricci, L., Testi, L., Natta, A., Neri, R., Cabrit, S., \& Herczeg, G. J. 2010, A\&A, 512, A 15

Robitaille, T. P., Whitney, B. A., Indebetouw, R., \& Wood, K. 2007, ApJS, 169, 328

Strom, K. M., Strom, S. E., Edwards, S., Cabrit, S., \& Skrutskie, M. F. 1989, AJ, 97, 1451

Tanaka, H., Himeno, Y., \& Ida, S. 2005, ApJ, 625, 414

van den Ancker, M. E., de Winter, D., \& Tjin A Djie, H. R. E. 1998, A\&A, 330, 145

Vinković, D., Ivezić, Ž., Jurkić, T., \& Elitzur, M. 2006, ApJ, 636, 348

Wheelwright, H. E., Oudmaijer, R. D., \& Goodwin, S. P. 2010, MNRAS, 401, 1199 